$$
\begin{aligned}
& x=0, \quad v=w=0, \quad x=l, \quad \partial^{2} v / \partial x^{2}=\partial^{2} w / \partial x^{2}=0 \\
& \partial v / \partial x=\hat{\partial} w / \partial x=0, \quad \quad \partial^{\mathrm{s}} v / \partial x^{8}=\partial^{\mathrm{s}} w / \partial x^{3}=0 \\
& \varphi=0, \quad \partial_{\varphi} / \partial x=0
\end{aligned}
$$

この場合紙面の都合上詳細な計算は省略するが，固有 値はそれぞれつぎのようになる。

$$
\begin{aligned}
& \kappa_{m v} \mathrm{I}=\kappa_{1 w^{(0)}}\left[\left\{\left(\frac{5}{4}+2 \mu\right)-\left(\frac{1}{2}+\mu\right) \sqrt[4]{\delta \kappa_{m y}}\right.\right. \\
& \times \frac{\cosh \sqrt[4]{\delta \kappa_{m o}}+\cos \sqrt[4]{\delta \kappa_{m v}{ }^{0}}}{\sinh \sqrt[4]{\delta \kappa_{m v}}+\sin \sqrt[4]{\delta \kappa_{m v}{ }^{0}}} \\
& +\left(\frac{1}{3}+\frac{\mu}{2}\right) \sqrt{\delta \kappa_{m v^{0}}}
\end{aligned}
$$

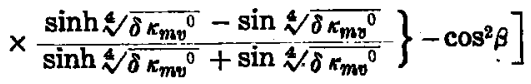

$$
\begin{aligned}
& \text { したがっ } \\
& \kappa_{1 v} \mathrm{I}=\kappa_{1 w}{ }^{(0)}\left[(1.19334+1.57088 \mu)-\cos ^{2} \beta\right] \\
& \kappa_{2 v} \mathrm{I}=\kappa_{1+v}{ }^{(0)}\left[(6.4782+8.6471 \mu)-\cos ^{2} \beta\right] \\
& \kappa_{3 v} \mathrm{I}=\kappa_{1 w}{ }^{(0)}\left[(17.8595+24.9521 \mu)-\cos ^{2} \beta\right] \\
& \text { また } \kappa_{1 w}, \kappa_{2 w w}, \kappa_{1 \varphi} \text { はそれぞれ } \\
& \kappa_{1 w v}=\left[(1.875104)^{4}-57.74443 \sigma^{2}\right] \\
& +\lambda\left\{1.19334+1.57088 \mu-\sin ^{2} \beta+\sigma^{2}[-1.61621\right. \\
& \left.\left.-0.857308 \mu-23.0596 \sin ^{2} \beta\right]\right\} /\left\{1+\sigma^{2}[23.0596\right. \\
& +23.3263 \nu]\}
\end{aligned}
$$

$$
\begin{aligned}
& \kappa_{2 w}=\left[(4.69409)^{4}-17676.2 \sigma^{2}\right]+\{\lambda 6.4782 \\
& +8.6471 \mu-\sin ^{2} \beta+\sigma^{2}[-939.104 \\
& \left.\left.-1452.27 \mu+164.101 \sin ^{2} \beta\right]\right\} /\left\{1+\sigma^{2}\right. \\
& {[-164.101+2469.56 \nu]} \\
& \kappa_{1 \varphi}=\left[(7.04790)^{4}+25688.8 \sigma^{2}\right] \\
& +\lambda\left\{\sigma^{2}[0.908031+1.11856 \mu\right. \\
& \left.\left.-0.0175080 \sin ^{2} \beta\right]\right\} /\left\{0.5 \nu+\sigma^{2}(0.0175080\right. \\
& -3.52124 \nu)\} \\
& \text { ここで, 計算の便宜上, } \varepsilon=1 \text { ととっている. } \\
& \text { 以上の結果より，断面の重心と茨り中心が一致しな } \\
& \text { いことが振動数に及湆す影響は翼のアスペクト比が小 } \\
& \text { さい場合に大きくなり，また低次振動より高次振動の } \\
& \text { 方が大きいようである。なお最初に記した様に実際の } \\
& \text { 回転翼は幾何学的に据れた形状をもっておりこのこと } \\
& \text { も振動数に影暜を及ぼすが，これについては別の機会 } \\
& \text { にゆずることとする。 }
\end{aligned}
$$

$$
\text { 文献 }
$$

1) K. Sezawa and I. Utida: On the Frequencies of Flexural Vibration of a Rotating Propeller Blade. J. Aero. Reg. Inst., Tokyo Univ.. No. 181. (1939).

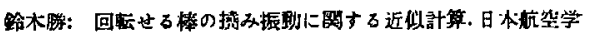

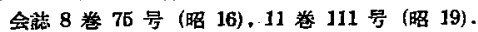

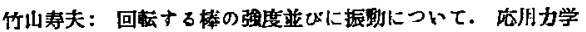
1 卷 4.5 号 (1948), 2 卷 10 号 (1949),

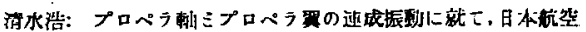
学会视 11 卷 105 是 (嘫 19),

H. La and J. L. Renbarger: Bending Vibrations. of a Rotating Beam. Proc. lst U. S. National Congress of A pp. Mech. (1951).

2) (1) 参照.

\title{
Note on the Flow just behind Three-Dimensional Shock ${ }^{*}$
}

\author{
By Shigeki MoRIOKA**
}

\begin{abstract}
The concise expressions which determine the derivatives of flow quantities just behind a steady three dimensional shock with respect to the space coordinates, are generally derived according to Thomas' procedure, under the assumption of regularity of shock surface. These expressions assume comparatively simple froms when we take the length of the lines of curvature as the parameters. As special cases, they are related to the flow just behind two-dimensional or axially symmetrical shock. The consistency relation is derived for axially symmetrical shock. Then it is is the same as shown that the distribution of singular shock directions for axially symmetrical shock that for two-dimensional shock.
\end{abstract}

\section{Introduction}

The concise expressions which determine the derivatives of velocity components, density and

- Received, May 17 th, 1956.

** Yoshida College, University of Kyoto. pressure just behind a steady two-dimensional shock with respect to space coordinates, have been provided by T. Y. Thomas' ${ }^{1}$, under the assumption of regularity of shock surface. Those are necessary to determine, for instance, the direction 
of the equi-speed line, the equi-density line, etc., their curvature just behind shock, or the direction of spines in the hodograph plane, or to attempt the expansion of flow quantities from the shock. Further, using those results, he ${ }^{2)}$ has given the consistency relation that connects the curvature of stream line just behind shock and its derivatives along the stream line with the curvature of the shock line and its derivatives along shock. Then he has investigated the distribution of singular shock direction. S. F. Shen ${ }^{8)}$ has discussed this in detail.

In this paper, the general procedure is provided that extends those expressions to the flow just behind three dimensional steady shock. When we take the length of lines of curvature as parameters, we obtain comparatively simple expressions. As special cases, they are related to the flow just behind two-dimensional or axially symmetrical shock")

If the problem is restricted to axially symmetrical shock from the first, the treatment becomes more simple. The consistency relation is derived for axially symmetrical shock. Then it is shown that the distribution of singular shock directions for axially symmetrical shock is the same as that in the two-dimensional case.

These expressions are applicable to the flow of steady, ideal gas just behind a shock by neglecting viscosity and heat conductivity.

\section{Fundamental relations}

Let $\phi$ and $\phi$ be two independent parameters, then, a shock surface is expressed by position vector,

$$
x=x(\phi, \psi)=x_{\alpha} e_{\alpha},
$$

where $e_{a}(a=1,2,3)$ are spacial fundamental vectors. Here the summation convention is used and this convention will be used throughout this paper. When we consider $\psi=$ constant and $\phi=$ constant, we call them $\phi$-curve and $\psi$-curve, respectively, and generally we call them parameter curves.

At a point $(\phi, \psi)$ on a shock surface, the tangent vectors to $\phi-$ and $\phi$-curve are expressed as

$$
\frac{\partial x}{\partial \phi}=x, \phi=\frac{\partial x_{\alpha}}{\partial \phi} e_{\alpha}, \quad \frac{\partial x}{\partial \phi}=\dot{x},{ }_{\psi}=\frac{\partial x_{\alpha}}{\partial \phi} e_{\alpha},
$$

The unit vector of surface normal in this point is expressedzas,

$$
\nu=\frac{x, \phi x, \psi}{\sqrt{(x, \phi \times x, \psi)^{2}}} .
$$

Here, we assume that $x, \phi \times x, \psi \neq 0$.

Let, $x^{2}{ }_{, \phi}=E, x, \phi x, \psi=F, x^{2}, \psi=G$, and let $\lambda$ and $\mu$ be tangent unit vectors to $\phi$ - and $\psi$-curve, respectively. Then,

$$
\begin{aligned}
& \lambda=\lambda_{\alpha} e_{\alpha}=\frac{1}{\sqrt{\mathscr{E}}} x, \quad \mu=\mu_{\alpha} e_{\alpha}=\frac{1}{\sqrt{G}} x, \phi \\
& \nu=\nu_{\alpha} e_{\alpha}=\frac{1}{\sqrt{E} G-F^{2}}(x, \phi \times x, \psi) .
\end{aligned}
$$

Among $\lambda, \mu$ and $\nu$, there exist the following relations:

$$
\begin{aligned}
\lambda \cdot \nu=\lambda_{\alpha} \nu_{\alpha}=0, \quad \mu \cdot \nu=\mu_{\alpha} \nu_{\alpha} & =0, \\
\lambda \cdot \mu=\lambda_{\alpha} \dot{\mu}_{\alpha}=\frac{F}{\sqrt{\bar{D} G}}, \quad \lambda \times \mu & =\frac{\sqrt{E G-F^{2}}}{\sqrt{\overline{E G}} \nu} \\
& =e_{\alpha}\left(\lambda_{B} \cdot \mu_{T}\right) .
\end{aligned}
$$

Let $u_{1}=u_{1 \alpha} e_{\alpha}, p_{1}$ and $\rho_{1}$ be velocity, pressure and density of the flow in front of the shock, and let $u=u_{\alpha} e_{\alpha}, p, \rho$ be ones just behind shock. Let the velocity components to surface normal, tangent to $\phi$ - and $\phi$-curve distinct by suffixes $n$, $\lambda$ and $\mu$, respectively. Then,

$$
\begin{aligned}
& u_{1 n}=u_{1} \cdot \nu=u_{1 \alpha} \nu_{\alpha}=\frac{\sqrt{E G}}{\sqrt{E \bar{G}-F^{2}}} u_{1 \alpha}\left(\lambda_{\beta} \mu_{\gamma}\right), \\
& u_{n}=u \cdot \nu=\mu_{\alpha} \nu_{\alpha}=\frac{\sqrt{E G}}{\sqrt{E G-F^{2}}} u_{\alpha}\left(\lambda_{\beta} \mu_{\Upsilon}\right), \\
& u_{\lambda}=u \cdot \lambda=u_{\alpha} \lambda_{\alpha}, \quad u_{\mu}=u \cdot \mu=u_{\alpha} \mu_{\alpha} .
\end{aligned}
$$

According to Thomas' expression, the shock conditions and the differential equations of flow are written as follows:

$$
\begin{aligned}
& u_{\alpha}=u_{1 \alpha}-\frac{2\left(\rho_{1} u_{1 n}^{2}-r p_{1}\right) \nu_{\alpha}}{(r+1) \rho_{1} u_{1 n}}, \\
& p=p_{1}+\frac{2\left(\rho_{\alpha} u_{1 n}{ }^{2}-r p_{1}\right)}{r+1}, \\
& \rho=\rho_{1}+\frac{2 \rho_{1}\left(\rho_{1} u_{1 n}{ }^{2}-r p_{1}\right)}{2 r p_{1}+(r-1) \rho_{1} u_{1 n^{2}}}, \\
& p,_{\alpha}+\rho u_{\sigma} u_{\alpha, \sigma}=0, \\
& \rho, \alpha u_{\alpha}+\rho u_{\sigma, \sigma}=0, \\
& \rho u_{a, \beta} u_{\alpha} u_{\beta}-r p u_{\sigma, \sigma}=0 .
\end{aligned}
$$

\section{First derivatives of flow quantities}

If we partially differentiate the shock conditions (3), (4) and (5) with respect to $\phi$ or $\psi$, we obtain

$$
\left.\begin{array}{ll}
u_{\alpha, \beta} \lambda_{\beta}=\frac{1}{\sqrt{E}} A_{\alpha \beta,}, & p_{, \beta} \lambda_{B}=\frac{1}{\sqrt{E}} B_{\beta,} \\
\rho, \beta, \lambda_{B}=\frac{1}{\sqrt{E}} C_{\phi,} & u_{\alpha, \beta} \mu_{\beta}=\frac{1}{\sqrt{G}} A_{\alpha \psi,} \\
p_{, \beta} \mu_{\beta}=\frac{1}{\sqrt{G}} B_{\psi,} & \rho_{, \beta} \mu_{\beta}=\frac{1}{\sqrt{G}} C_{\psi,}
\end{array}\right\}
$$


where the quantities indicated in the right hand side; $A_{\alpha \phi,} B_{\phi}, \cdots e t c .$, are obtained by differentiating the quantities of right hand side of Eqs. (3), (4) and (5) with respect to $\phi$ or $\phi$.

We define the quantity $\xi_{i}^{\alpha}$ by the matrix form as

$$
\left[\xi_{1}^{\alpha}\right]=\left[\begin{array}{ccc}
\xi_{1}^{1} & \xi_{1}^{2} & \xi_{1}^{8} \\
\xi_{2}{ }^{1} & \xi_{2}^{2} & \xi_{2}{ }^{3} \\
\xi_{3}^{1} & \xi_{3}{ }^{2} & \xi_{3}^{8}
\end{array}\right]=\left[\begin{array}{lll}
\lambda_{1} & \lambda_{2} & \lambda_{3} \\
\mu_{1} & \mu_{2} & \mu_{3} \\
u_{1} & u_{2} & u_{3}
\end{array}\right] .
$$

Then, its determinant is $\left|f_{i}^{\alpha}\right|=u_{\alpha}\left(\lambda_{\theta} \mu_{r}\right)=$ $\left(\sqrt{E G-\overline{F^{2}}} / \sqrt{E \bar{G}}\right) u_{n}$.

Let the inverse matrix of $\left[\xi_{i}^{\alpha}\right]$ be $\left[\zeta_{\alpha}{ }^{l}\right]$, then we obtain

$$
\begin{aligned}
& {\left[\zeta_{a}{ }^{4}\right]=\left[\begin{array}{ccc}
\zeta_{1}^{1} & \zeta_{1}^{2} & \zeta_{1}^{8} \\
\zeta_{2}{ }^{1} & \zeta_{2}^{2} & \zeta_{2}^{3} \\
\zeta_{3}{ }^{1} & \zeta_{3}{ }^{2} & \zeta_{3}^{8}
\end{array}\right]=\frac{\sqrt{E G}}{\sqrt{E G-F^{2}}} \frac{1}{u_{n}}} \\
& \times\left[\begin{array}{lll}
\left(\mu_{2} u_{3}-\mu_{3} u_{2}\right) & \left(\lambda_{3} u_{2}-\lambda_{2} u_{3}\right) & \left(\lambda_{2} \mu_{3}-\lambda_{3} \mu_{2}\right) \\
\left(\mu_{3} u_{1}-\mu_{1} u_{3}\right) & \left(\lambda_{1} u_{3}-\lambda_{3} u_{1}\right) & \left(\lambda_{3} \mu_{1}-\lambda_{1} \mu_{3}\right) \\
\left(\mu_{1} u_{2}-\mu_{2} u_{1}\right) & \left(\lambda_{2} u_{1}-\lambda_{1} u_{2}\right) & \left(\lambda_{1} \mu_{2}-\lambda_{2} \mu_{1}\right)
\end{array}\right]
\end{aligned}
$$

From the shock conditions (9) and the differential equations of flow (6), (7) and (8), we obtain

$$
\begin{aligned}
& u_{\alpha, \beta} \xi_{i}^{\alpha} \xi_{j}^{\beta}=A_{i j}, \\
& \rho, \beta \xi \xi^{\beta}=a_{i},
\end{aligned}
$$

where

$$
\begin{array}{ll}
A_{11}=\frac{1}{\sqrt{E}} A_{\alpha \phi} \lambda_{\alpha}, & A_{12}=\frac{1}{\sqrt{G}} A_{\alpha \psi} \lambda_{\alpha}, \\
A_{13}=-\frac{1}{\sqrt{E}} \frac{B_{\phi}}{\rho}, & A_{21}=\frac{1}{\sqrt{E}} A_{\alpha \phi} \mu_{\alpha}, \\
A_{22}=\frac{1}{\sqrt{G}} A_{\alpha \psi} \mu_{\alpha}, & A_{28}=-\frac{1}{\sqrt{G}} \frac{B_{\psi}}{\rho}, \\
A_{31}=\frac{1}{\sqrt{E}} A_{\alpha \phi} u_{\alpha}, & A_{32}=\frac{1}{\sqrt{G}} A_{\alpha \psi} u_{\alpha}, \\
A_{33}=\frac{r p}{\rho} u_{\sigma, \sigma}=c^{2} u_{\sigma, 0} .
\end{array}
$$

Thus, the first derivatives of velocity components and density just behind shock can be expressed as

$$
\begin{aligned}
& u_{\alpha, \beta}=A_{i i} \zeta_{\alpha^{i}} \zeta_{\beta}^{\prime}, \\
& \rho, \beta=a_{b} \zeta_{\beta}^{6} .
\end{aligned}
$$

While the derivatives of pressure can be obtained from $\mathrm{Eq}$. (6) and above results,

$$
p,_{\beta}=-\rho A_{t 3} \zeta_{\alpha}{ }^{i} \text {. }
$$

Among the above expressions, all the coefficients, except $A_{33}$ and $a_{3}$, have the permitted determination, in the sense that these quantities can be expressed by the conditione in front of shock and the form of the shock surface. While $A_{33}$ and $a_{3}$ can be expressed by other coefficients.

4. Computation of coefficients $A_{33}$ and $a_{3}$.

By Weingarten's formula,

$$
\begin{aligned}
& \frac{\partial \nu}{\partial \phi}=a x,_{\phi}+b x_{\psi}=\sqrt{E} a \lambda+\sqrt{G b} \mu \\
& \frac{\partial \nu}{\partial \psi} a^{\prime} x_{, \phi}+b^{\prime} x_{, \psi}=\sqrt{E^{\prime}} a^{\prime} \lambda+\sqrt{G} b \mu
\end{aligned}
$$

where

$$
\begin{array}{ll}
a=\frac{F M-G L}{E G-F^{2}}, & b=\frac{F L-E M}{E G-F^{2}}, \\
a^{\prime}=\frac{F N-G M}{E G-F^{2}}, & b^{\prime}=\frac{F M-E N}{E G-F^{2}},
\end{array}
$$

and $L=\nu \cdot x, \phi \phi, M=\nu \cdot x, \phi \psi, N=\nu \cdot x, \psi \psi$.

Thus, from Eqs. (1) and (2), we obtain

$$
\begin{aligned}
& \frac{\partial u_{1 n}}{\partial \phi}=\sqrt{E} a u_{\lambda}+\sqrt{G} b u_{\mu}, \\
& \frac{\partial u_{1 n}}{\partial \phi}=\sqrt{E} a^{\prime} u_{\lambda}+\sqrt{\bar{G}} b^{\prime} u_{\mu} .
\end{aligned}
$$

Here the problem is restricted to the case of steady shock, and it is assumed that $u_{1 \alpha}, p_{1}$ and $\rho_{1}$ are constant.

Differentiating partially the right hand side quantities of Eq. (4) with respect to $\phi$ or $\phi$, we obtain

$$
\begin{aligned}
& B_{\varnothing}=\frac{4 \rho_{1} u_{1 n}}{r+1}\left(\sqrt{ } E a u_{\lambda}+\sqrt{G} b u_{\mu}\right), \\
& B_{\Psi}=\frac{4 \rho_{1} u_{1 n}}{r+1}\left(\sqrt{E} a^{\prime} u_{\lambda}+\sqrt{G} b^{\prime} u_{\mu}\right) .
\end{aligned}
$$

Similary, from Eq. (3) :

$$
\begin{aligned}
A_{\alpha \phi}= & -\frac{B_{\phi \nu_{\alpha}}}{\rho_{1} u_{1 n}}+\frac{[\rho]}{\rho}\left\{\left(\sqrt{ } E a u_{\lambda}+\sqrt{G} b u_{\mu}\right) \nu_{\alpha}\right. \\
& \left.-\sqrt{ } E a u_{1 n} \lambda_{\alpha}-\sqrt{ } G b u_{1 n} \mu_{\alpha}\right\}, \\
A_{\alpha \psi}= & -\frac{B_{\psi} \nu_{\alpha}}{\rho_{1} u_{1 n}}+[\rho]-\left[\left(\sqrt{E} a^{\prime} u_{\lambda}+\sqrt{G} b^{\prime} u_{\mu}\right) \nu_{\alpha}\right. \\
& \left.-\sqrt{ } E a^{\prime} u_{1 n} \lambda_{\alpha}-\sqrt{G} b^{\prime} u_{1 n} \mu_{\alpha}\right\},
\end{aligned}
$$

where $[\rho]=\rho-\rho_{1}$, and from Eq. (5):

$$
\begin{aligned}
C_{\phi} & =\frac{-2}{(r+1) u_{n}}\{(r-1) \rho \\
& \left.-(r+1) \rho_{1}\right\}\left(\sqrt{E} a u_{\lambda}+\sqrt{G} b u_{\mu}\right), \\
C_{\psi} & =\frac{-2}{(r+1) u_{n}}\{(r-1) \rho \\
& \left.-(r+1) \rho_{1}\right\}\left(\sqrt{ } E a^{\prime} u_{\lambda}+\sqrt{G} b^{\prime} u_{\mu}\right) .
\end{aligned}
$$

Multiplying $\lambda_{a}$ or $\mu_{\alpha}$ to Eq. (18) and summing up with $\alpha$, we obtain

$$
\left.\begin{array}{l}
A_{\alpha \phi} \lambda_{\alpha}=-\frac{[\rho]}{\rho} \frac{1}{\sqrt{E}}(E a+F b) u_{1 n}, \\
A_{\alpha \phi} \mu_{\alpha}=-\frac{[\rho]}{\rho} \frac{1}{\sqrt{G}}(F a+G b) u_{1 n},
\end{array}\right\}
$$


Similary, from Eq. (19):

$$
\begin{aligned}
& A_{\alpha \psi} \lambda_{x}=-\frac{[\rho]}{\rho} \underset{\sqrt{ } E}{1}\left(E a^{\prime}+F b\right) u_{1 n}, \\
& \left.A_{\alpha \psi} \mu_{x}=-\frac{[\rho]}{\rho} \frac{1}{\sqrt{ } G}\left(F a^{\prime}+G b^{\prime}\right) u_{1 n} .\right\}
\end{aligned}
$$

Finally multiplying $u_{\alpha}$ to Eqs. (18) and (17) and summing up with $\alpha$ we obtain

$$
\begin{aligned}
A_{\alpha \phi} u_{\alpha}= & -\left(\frac{4}{r+1}+\frac{[\rho]^{2}}{\rho_{1} \rho}\right)\left(\sqrt{E} a u_{\lambda}\right. \\
& \left.+\sqrt{ } G b u_{\mu}\right) u_{n}, \\
A_{x \psi} u_{\alpha}= & -\left(\frac{4}{r+1}+\frac{[\rho]^{2}}{\rho_{1} \rho}\right)\left(\sqrt{ } E a^{\prime} u_{\lambda}\right. \\
& +\sqrt{ } \bar{G}^{\left.b^{\prime} u_{\mu}\right) u_{n} .}
\end{aligned}
$$

Using the relations (16) to (24), the coefficients of the right hand side of Eq. (12) can be determined, except $A_{33}$ and $a_{3}$, as follows:

$$
\begin{aligned}
& A_{11}=-\frac{[\rho]}{\rho_{1}} \frac{E a+F b}{E} u_{n}, \\
& A_{12}=-\frac{[\rho]}{\rho_{1}} \frac{E a^{\prime}+F b^{\prime}}{\sqrt{E G}} u_{n}, \\
& A_{13}=-\frac{4}{r+1} \frac{\sqrt{E} a u_{\lambda}+\sqrt{G} b u_{\mu}}{\sqrt{ } E} u_{n}, \\
& A_{21}=-\frac{[\rho]}{\rho_{1}} \frac{F a+G b}{\sqrt{E G}} u_{n}, \\
& A_{22}=-\frac{[\rho]}{\rho_{1}} \frac{F a^{\prime}+G b^{\prime}}{G} u_{n}, \\
& A_{23}=-\frac{4}{r+1} \frac{\sqrt{E} a^{\prime} u_{\lambda}+\sqrt{G} b^{\prime} u_{\mu}}{\sqrt{ } G} u_{n}, \\
& A_{31}=-\left(\frac{4}{r+1}+-\frac{[\rho]^{2}}{\rho_{1} \rho}\right) \\
& \quad \times \frac{\sqrt{E} a u_{\lambda}+\sqrt{G} b u_{\mu}}{\sqrt{ } E} u_{n}, \\
& A_{32}=-\left(\frac{4}{r+1}+\frac{[\rho]^{2}}{\rho_{1} \rho}\right) \\
& \times \frac{\sqrt{E} a^{\prime} u_{\lambda}+\sqrt{ } G b^{\prime} u_{\mu}}{\sqrt{ } G} u_{n},
\end{aligned}
$$

Now we notice that $\zeta \sigma^{1}, \zeta \sigma^{2}$ and $\zeta \sigma^{3}$ have expressed the components of vector $\left(\sqrt{E} \bar{G} / \sqrt{E G-F^{2}}\right)$ $\left(1 / u_{n}\right) \cdot(\boldsymbol{\mu} \times \boldsymbol{u}), \quad-\left(\sqrt{ } \boldsymbol{E} G / \sqrt{ } \boldsymbol{E} G-F^{2}\right)\left(1 / u_{n}\right) \cdot(\lambda \times$ $\boldsymbol{u}),\left(\sqrt{E} G / \sqrt{E} \bar{G}-\bar{F}^{2}\right)\left(1 / u_{n}\right) \cdot(\lambda \times \mu)$, hence

$$
\begin{aligned}
& \zeta_{\sigma}^{1} \zeta_{\sigma}^{1}=\frac{E G}{E G-F^{2}} \frac{u^{2}-u_{\mu}^{2}}{u_{n}^{2}}, \\
& \zeta_{\sigma}^{2} \zeta_{\sigma}^{2}=\frac{E G}{E G-F^{2}} \frac{u^{2}-u_{\lambda}^{2}}{u_{n}^{2}}, \quad \zeta_{\sigma}^{3} \zeta_{\sigma}^{8}=\frac{1}{u_{n}^{2}}, \\
& \zeta_{\sigma}^{1} \zeta_{\sigma}^{2}=\frac{E G}{E G-F^{2}} \cdot \frac{1}{u_{n}^{2}}\left(u_{\lambda} u_{\mu}-\frac{F}{\sqrt{E G}} u^{2}\right), \\
& \zeta_{\sigma}^{1} \zeta_{\sigma}^{3}=\frac{E G}{E G-F^{2}} \frac{1}{u_{n}^{2}}\left(\frac{F}{\sqrt{E G}} u_{\mu}-u_{\lambda}\right),
\end{aligned}
$$

$$
\zeta_{\sigma}^{2} \zeta_{\sigma}^{8}=\frac{E G}{E G-F^{2}}-\frac{1}{u_{n}^{2}}\left(\frac{F}{\sqrt{E G}} u_{\lambda}-u_{\mu}\right),
$$

Thus, inserting these relations into $A_{33}=c^{2} u_{\sigma, \sigma}$ $=c^{2} A_{i j} \zeta_{\alpha}^{i} \zeta_{\beta}^{j}$, we obtain

$$
\begin{aligned}
A_{s 3} & =\frac{-u_{n}}{\left(\frac{u_{n}^{2}}{c^{2}}-1\right)} \frac{E G}{E G-F^{2}}\left[\left\{\begin{array}{l}
{[\rho]} \\
\rho_{1}
\end{array}\right.\right. \\
& \times \frac{E a+F b}{E}\left(u^{2}-u_{\mu}^{2}\right) \\
& +\frac{[\rho]}{\rho_{1}} \frac{F a+G b}{\sqrt{E G}}\left(u_{\lambda} u_{\mu}-\frac{F}{\sqrt{E G}} u^{2}\right) \\
& +\left(\frac{8}{r+1}+\frac{[\rho]^{2}}{\rho_{1} \rho}\right)\left(\frac{\sqrt{E} a u_{\lambda}+\sqrt{ } G b u_{\mu}}{\sqrt{E}}\right) \\
& \left.\times\left(\frac{F}{\sqrt{E} \bar{G}} u_{\mu}-u_{\lambda}\right)\right\} \\
& +\left\{\frac{[\rho]}{\rho_{1}} \frac{E a^{\prime}+F b^{\prime}}{E}\left(u^{2}-u_{\lambda}^{2}\right)\right. \\
& +\frac{[\rho]}{\rho_{1}} \frac{F a^{\prime}+G b^{\prime}}{\sqrt{E G}}\left(u_{\lambda} u_{\mu}-\frac{F}{\sqrt{E G}} u^{2}\right) \\
& +\left(\frac{.8}{r+1}+\frac{[\rho]^{2}}{\rho_{1} \rho}\right)\left(\frac{\sqrt{E} a^{\prime} u_{\lambda}+\sqrt{G} b^{b^{\prime}} u_{\mu}}{\sqrt{ } G}\right) \\
& \left.\left.\times\left(\frac{F}{\sqrt{E} \bar{G}} u_{\lambda}-u_{\mu}\right)\right\}\right] .
\end{aligned}
$$

While $a_{i}$ can be expressed as follows:

$$
\begin{aligned}
a_{1}= & \frac{-2}{(r+1) u_{n}}\{(r-1) \rho \\
& \left.-(r+1) \rho_{1}\right\} \frac{\sqrt{E} a u_{\lambda}+\sqrt{G} b u_{\mu}}{\sqrt{ } E}, \\
a_{2}= & -\frac{-2}{(r+1) u_{n}}\{(r-1) \rho \\
& \left.-(r+1) \rho_{1}\right\} \frac{\sqrt{E} a^{\prime} u_{\lambda} \mid+\sqrt{G} b^{\prime} u_{\mu}}{\sqrt{G}}, \\
a_{3}=- & -\frac{\rho}{c^{2}} A_{33} .
\end{aligned}
$$

Now if we choose two Cartessian coordinates $x_{1}$ and $x_{2}$ as parameters $\phi$ and $\phi$, and if we assume that shock surface can be expressed by $x_{3}=x_{3}$ $\left(x_{1}, x_{2}\right)$, and let be $\partial x_{3} / \partial x_{1}=p, \partial x_{3} / \partial x_{2}=q, \partial^{2} x_{3} /$ $\partial x_{2}{ }^{2}=r, \partial^{2} x_{3} / \partial x_{1} \partial x_{2}=s, \partial^{2} x_{3} / \partial x_{2}{ }^{2}=t$, then, we obtain

$$
\begin{aligned}
& E=1+p^{2}, \quad F=p q, \quad G=1+q^{2}, \\
& L=\frac{r}{\sqrt{1+p^{2}+q^{2}}}, \quad M=\frac{s}{\sqrt{1+p^{2}+q^{2}}}, \\
& N=\frac{t}{\sqrt{1+p^{2}+q^{2}}},
\end{aligned}
$$

While, if we choose the length of lines of curvature as parameters, we obtain $E=G=1$, and at a point except umbilic point (i. e. $L: M: N \neq$ 
$E: \dot{F}: G), F=M=0, L=\kappa_{\lambda}$ and $N=\kappa_{\mu}$, where $\kappa_{\lambda}$ and $\kappa_{\mu}$ are the normal curvature for $\phi$-and $\psi$-curve at the point considered. Hence,

$$
a=-\kappa_{\lambda}, \quad b=a^{\prime}=0, \quad b^{\prime}=-\kappa_{\mu} .
$$

Thus, in this case, we get the comparatively simple form for coefficients $A_{i j}$ and $a_{b}$ as

$$
\left.\begin{array}{rl}
A_{11}= & \frac{[\rho]}{\rho_{1}} u_{n} \kappa_{\lambda}, \quad A_{12}=0, \\
A_{13}= & \frac{4}{r+1} u_{n} u_{\lambda} \kappa_{\lambda}, \quad A_{21}=0, \\
A_{22}= & \frac{[\rho]}{\rho_{1}} u_{n} \kappa_{\mu}, \quad A_{23}=\frac{4}{r+1} u_{n} u_{\mu} \kappa_{\mu}, \\
A_{31}= & \left(\frac{4}{r+1}+\frac{[\rho]^{2}}{\rho_{1} \rho}\right) u_{n} u_{\lambda} \kappa_{\lambda}, \\
A_{32}= & \left(\frac{4}{r+1}+\frac{[\rho]^{2}}{\rho_{1} \rho}\right) u_{n} u_{\mu} \kappa_{\mu}, \\
A_{33}= & \frac{n_{n}}{\frac{u_{n}^{2}}{c^{2}}-1}\left[\left\{\frac{[\rho]}{\rho_{1}}\left(u^{2}-u_{\lambda}^{2}\right)\right.\right. \\
& \left.-\left(\frac{8}{r+1}+\frac{[\rho]^{2}}{\rho_{1} \rho}\right) u_{\lambda}^{2}\right\} \kappa_{\lambda} \\
& +\left\{\frac{[\rho]}{\rho_{1}}\left(u^{2}-u_{\mu}^{2}\right)\right. \\
& \left.\left.-\left(\frac{8}{r+1}+\frac{[\rho]^{2}}{\rho_{1} \rho}\right) u_{\mu}^{2}\right\} \kappa_{\mu}\right], \\
a_{1}= & \frac{2 u_{\lambda} \kappa_{\lambda}}{(r+1) u_{n}}\left\{(r-1) \rho-(r+1) \rho_{1}\right\}, \\
a_{2}= & \frac{2 u_{\mu} \kappa_{\mu}}{(r+1) u_{n}}\left\{(r-1) \rho-(r+1) \rho_{1}\right\}, \\
a_{3}= & -\frac{\rho}{c^{2}} A_{33} .
\end{array}\right\}
$$

\section{Curvature of stream line just behind shock}

If we indicate the length along a stream line by $\eta$, the curvature of stream line $K$ is defined as,

$$
K^{2}=\frac{\partial^{2} x}{\partial \eta^{2}} \frac{\partial^{2} x}{\partial \eta^{2}},
$$

namely, $K$ is the magnitude of vector $\partial^{2} x / \partial \eta^{2}$. Now, taking account of $\partial x / \partial \eta=\left(u_{\alpha} / u\right) e_{\alpha}$ and $u_{\alpha} u_{\alpha}=u^{2}$,

$$
K^{2}=\frac{1}{u^{2}}\left\{\frac{\partial u_{\alpha}}{\partial \eta} \frac{\partial u_{\alpha}}{\partial \eta}-\left(\frac{\partial u}{\partial \eta}\right)^{2}\right\} .
$$

Further, since $\left(\partial u_{\alpha} / \partial_{\eta}\right)\left(\partial u_{\alpha} / \partial \eta\right)=\left(1 / u^{2}\right)\left(u_{\alpha, \beta} u_{\beta}\right)$ $\left(u_{\alpha, \beta} u_{\beta}\right), \partial u / \tilde{o}_{\eta}=\left(1 / u^{2}\right) u_{\alpha, \beta} u_{\alpha} u_{\beta}$, and $u_{\alpha, \beta} u_{\beta}=$ $A_{i 3} \zeta_{\alpha}{ }^{4}, u_{\alpha, \beta} u_{\alpha} u_{\beta}=A_{a z}$,

$$
K^{2}=\frac{1}{u^{4}}\left\{\left(A_{i 8} \zeta_{\alpha}^{9}\right)\left(A_{i s} \zeta_{\alpha}\right)-\frac{1}{u^{2}} A_{28}^{2}\right\} .
$$

In particular, if we choose the length of lines of curvature as parameters,

$$
\begin{aligned}
K^{2} & =\frac{1}{u^{4} u_{n}^{2}}\left\{\left(A_{13} u-A_{33} \frac{u_{\lambda}}{u}\right)^{2}\right. \\
& \left.+\left(A_{23} u-A_{33} \frac{u_{\mu}}{u}\right)^{2}-\left(A_{13} u_{\mu}-A_{23} u_{\lambda}\right)^{2}\right\}
\end{aligned}
$$

where $A_{13}, A_{23}$ and $A_{33}$ are obtained by Eqs. (29).

In the case of two dimensional shock, it is assumed that $\kappa_{\mu}$ and $u_{\mu}$ (or $\kappa_{\lambda}$ and $u_{\lambda}$ ) vanish identically. Thus, we have

$$
K^{2}=\frac{1}{u^{4} u_{n}^{2}}\left(A_{13} u-A_{33} \frac{u_{\lambda}}{u}\right)^{2},
$$

where $A_{13}=\{4 /(r+1)\} u_{n} u_{\lambda} \kappa_{\lambda}, A_{33}=\left\{u_{n} /\left(u_{n}{ }^{2} / c^{2}-\right.\right.$ 1) $\} \cdot\left\{\left([\rho] / \rho_{1}\right) u^{2}-\left\{8 /(\gamma+1)+\left([\rho]^{2} / \rho_{1} \rho\right) u_{\lambda}^{2}\right\} \kappa_{\lambda}\right.$,

This is the result derived by Thomas.

In the case of axially symmetrical shock, the lines of curvature are intersections of the shock surface with the meridian plane and perpendicular to the axis. We choose them $\phi$ - and $\psi$-curve, respectively. Then $u_{\mu}=0$ and hence $A_{23}=0$. Thus

$$
K^{2}=\frac{1}{u^{4} u_{n 2}^{2}}\left(A_{13} u-A_{33} \frac{u_{\lambda}}{u}\right)^{2},
$$

where $A_{13}=\{4 /(\gamma+1)\} u_{n} u_{\lambda} \kappa_{\lambda}, \quad A_{33}=\left\{u_{n} /\left(u_{n}^{2} / c^{2}\right.\right.$ $-1)\} \cdot\left[\left\{[\rho] / \rho_{1}\right) \cdot u^{2}-\left(8 /(\gamma+1)+\left([\rho]^{2} / \rho_{1} \rho\right) u_{\lambda}^{2}\right)\right\} \kappa_{\lambda}$ $\left.+\left([\rho] / \rho_{1}\right)\left(u^{2}-u_{\lambda}^{2}\right) \kappa_{\mu}\right]$. Let $d$ and $\alpha$ be radii of $\psi$-curve (circle) and the shock angle at the point considered, respectively. Then $\kappa_{\mu}=(1 / d) \cos \alpha$.

When we define the curvature as positive or negative according as the curve is concave upward or downward, respectively, the expression for $K$ must take the negative root.

6. Singular shock directions for axially symmetrical shock

If the problem is restricted to axially symmetrical shock from the first, the treatment is very simple. In this case, the differential equations of flow can be written as follows:

$$
\left.\begin{array}{l}
p_{\alpha}+\rho u_{\sigma} u_{\alpha, \sigma}=0, \\
\rho u_{\alpha}+\rho u_{\sigma, \sigma}+\rho \frac{\bar{u}}{\bar{x}}=0, \\
\rho u_{\alpha, \beta} u_{\alpha} u_{\beta}-\gamma p\left(u_{\sigma, \sigma}+\frac{\bar{u}}{x}\right)=0,
\end{array}\right\}
$$

where $\bar{x}$ is the distance from the symmetrical axis to the point considered, and $\bar{u}$ is velocity component perpendicular to this axis at this point and in these expressions the suffixes take only 1 
and 2. The shock conditions are the same as in the two-dimensional case.

Using rotated coordinates ${ }^{2}$ and taking into account only the terms which involve the highest derivative of velocity components, we have the same expression for $n$-th derivative of curvature of streamline just behind shock as in the two dimensional case, namely

$$
\frac{d^{n} K}{d \eta^{n}}=\frac{u_{2}, 11 \cdots 1}{v}
$$

By taking account of

$$
\left(\frac{1}{\bar{x}}\right)_{, \beta \cdots \tau}=\frac{\delta_{\beta} \cdots \delta_{\gamma}}{(-\sin \alpha)^{n}} \frac{d^{n} K_{\mu}}{d S^{n}},
$$

just behind shock, where $\delta_{1}=\sin \omega, \delta_{2}=\cos \omega, \omega$ is deflection angle and $s$ is arc length along the shock surface in a meridian plane, finally, $u_{2}, 11 \ldots 1$ can be determined by solving the following systems which are obtained from the differential equations of flow and the shock conditions :

$$
\begin{gathered}
\left(\frac{u^{2}}{c^{2}}-1\right) u_{1,1 \beta \cdots r}-u_{2,2 \beta} \cdots \gamma \\
=\bar{u} \frac{\delta_{\beta} \cdots \delta_{\gamma}}{(-\sin \alpha)^{n}} \frac{d^{n} K_{\mu}}{d S^{n}}, \\
u_{\alpha, 1 \beta \cdots \gamma} \lambda_{\alpha} \lambda_{\beta} \ldots \lambda_{r}=-\frac{B}{\rho u} \frac{d^{n} K_{\lambda}}{d S^{n}}, \\
u_{2, \alpha \beta \cdots \gamma} \lambda_{x} \lambda_{\beta} \ldots \lambda_{\gamma}=A_{2} \frac{d^{n} K_{\lambda}}{d S^{n}},
\end{gathered}
$$

The Theory of Hydrodynamic Stability: C. C. Lin, Cambridge, Cambridge 1955, 155 頁, 15 $\times 23 \mathrm{~cm}, 22 \mathrm{~s} .6 \mathrm{~d}$.

“流体力学的安定理論”について初の成書が Cambridge Monographs の一つとして現われた，著者 C. C. Lin はMIT の応用数学の教授で，安定理論に ついては戦時中加らまで数多くの指導的な仕事をし て来た人である。

本書で取扱われている安定理諭は，微小摫乱に対す る所謂線型安定理諭である。これは元来，層流から乱 ’流への遷移理諭の一部分をなすものであるが，今日で は具体的な結果を与えうる只一つの理論として，遷移 理諭の中でも独立した二つの体系を形造っている．前 世紀の末に始まる長い歴史をもつこの理論を，著者は かなり要領よくこの小册子にまとめている.

第 1 章 緒論：微小摬乱法による安定理論の定式化 と三三の興型的な流れに対する結果の概钼. 第 2 章 Couette 運動の安定性: Taylor の計算の紹介と実験 との比較。第 3 章 二次元 Poiseuille 流の安定性： 安定の充分条件についての古典的結果と, Heisenberg に始まる漸近解による固有値問題の解法とを述ぺる。 第 4 草 流体力学的安定の一般理論：不安定の動因を 遠心力などの外力によるもの（外力型）と粘性を媒介 とするむの（粘性型）とに分類し，各々について物理 的機棈を洘察する. 第 5 章 平板に沿弓境界層：実用 的に重要な圧樎性境界層の安定についてて, Lees, Lin, Dunn 等の計算を紹介する、第 6 章 他のほとんど平 行な流れ：境界層の安定に対する主流の乱れ等のパラ
Where $B$ and $A_{z}$ are same expressions as obtained by Thomas ${ }^{2)}$ for two dimensional case.

Thus, we prove the following consistency relation,

$$
\begin{aligned}
\frac{d^{n} K}{d \eta^{n}} & =G_{1 n}(M, \alpha) \frac{d^{n} K_{\lambda}}{d S^{n}} \\
& +G_{2 n}(M, \alpha) \frac{d^{n} K_{\mu}}{d S^{n}}+H_{n},
\end{aligned}
$$

where $H_{n}$ is the polynomial of $\kappa_{\lambda}, \kappa_{\mu}$ and their derivatives less than $n$-th order with respect to s. Further $G_{1 n}, G_{2 n}$ and coefficients of $H_{n}$ are rational functions of $M^{2}$ the coefficients of which are functions of $\alpha$, and both $G_{1 n}$ and $G_{2 n}$ have the zeros only in the subsonic region.

$d^{n} \kappa_{\mu} / d S^{n}$ can be determined by $\kappa_{\lambda}, \kappa_{\mu}$ and their derivatives less than $n$-th order and $G_{1 n}(\alpha, M)$ consist with two-dimensional one. Hence the singular direction where the power series approach based on Eq. (40) will fail, is given by the angle $\alpha$ at which any of $G_{1 n}$ 's vanish at given $M$, and its distribution is the same as two-dimensional one. But it has been assumed that $\kappa_{\mu}$ is finite.

\section{References}

1) T. Y. Thomas: J. Math. Phys., 26 (1947) 62.

2) T. Y. Thomas: J. Math. Phys., 28 (1949) 158

3) S. F. Shen: J. Math. Phys.. 81 (1952).

4) H. Oguchi: J. Phyg. Soc. Japan, 9 (1954) 861

\section{評 D}

メターの影響を調ぺ，助走流・混合流など境界層に類 似の流についての計算結果を述べる.第 7 章 天体及 び地球物理学的に興味ある安定問題の例：大気循環， 対流など外力型不安定の実例を簡単に述べる。第 8 章 平行流の安定の数学的議論：粘性型不安定に伴了数学 的問題, 殊に高 Reynolds 数に対する渐近解が多価 性をもつことによる困難の解決を， Lin, Wasaw， Morawetzの研究を中心として述へる。この章は第3 章と共に本書中の圧巻である，巻末の文献表は豊富す。 安定理論についてのほとんど全業績を尽している。

不安定の二つの型のうち，乱流への遷移に主として 関係するのは粘性型不安定であり，本書の記述の重点 もまたここに置かれている。たた，渐近解法について の詳しい議論が第 8 章まで後回しになってているか゚, こ れは第 3 章と一緒にしてもっと丁整に書いてくれた方 が有難かったと思う。他にも欲をいえば，叙述のキメ がかなり荒く，諭文から理届抜きで結果の式を引用し てあることの多い第 5 章以後など，読むのに相当骨が 折れる.これは本書の意図が，安定理論の応用例の稆 介よりも，理論そのむのの構造の解説にあると思われ るだけに残念である。また，初版の故ですあろうが愦 植か割合に多く，殊に式の中のミスはいささか読者泣 かせである。

とはいえ，内容的にも多岐に亘り，また厄介な数式 的議諭の欠かせない流体力学的安定理論を，ここまて まとめ上げた著者の腕は非凡である。この方面の最初 の専門書として関心ある諸氏の一珫をお勧めしたい。 (翼 友正) 